\title{
Miniaturisierter Sauerstoffsensor auf Festkörperelektrolytbasis für die in-situ Messung in der Atemgasanalyse
}

\author{
A. Günther ${ }^{1}$, R. Baumann ${ }^{1}$, T. Schmiel ${ }^{1}$, T. Tajmar ${ }^{1}$ \\ ${ }^{1}$ Institut f. Luft- u. Raumfahrttechnik, TU Dresden, 01307 Dresden, Germany; Tel.-Nr. +49 0351/463- \\ 38234, E-Mail: andreas.guenther1@tu-dresden.de
}

\begin{abstract}
Zusammenfassung
Der vorliegende Beitrag gibt einen zusammenfassenden Überblick über den Aufbau, die Funktion und das Messverhalten eines neuartigen elektrochemischen Sauerstoffsensors für den Einsatz in der medizinischen Atemgasanalyse. Hervorzuheben ist dabei vor allem der hohe Grad der Miniaturisierung und die robuste Bauweise des Sensors, die es ermöglicht, die Gasmessung direkt im Hauptatemstrom (in-situ) durchzuführen, wodurch auf externe Analysatoren verzichtet werden kann. Die Miniaturisierung führt zudem zu extrem niedrigen Ansprechzeiten, die vor allem für atemzugaufgelöste Messungen in der Leistungsdiagnostik (Spiroergometrie) notwendig sind. Messungen zeigen, dass die Anstiegs- und Abfallzeiten im Bereich von 80-120 ms erreicht werden können. Auch die Messgenauigkeit lässt sich bereits mit kommerziellen Systemen vergleichen. Für den Messbereich der Spiroergometrie $\left(10-21 \% \mathrm{O}_{2}\right)$ liegt die Messabweichung der vorgestellten Sensoren unter $\pm 0,15 \mathrm{Vol} . \%$, für den Bereich der Beatmungstechnik (21-100\%) bei $\pm 3 \%$ v.ME.
\end{abstract}

Keywords: Gassensorik, Atemgasanalyse, Hauptstrommessung, Miniaturisiert, Festkörperelektrolyt

\section{Einleitung}

Keramische Hochtemperatursensoren zur Messung des Sauerstoffpartialdrucks haben sich in den letzten Jahren verstärkt im Bereich der medizinischen Atemgasanalyse etabliert. Neben konkurrierenden Technologien wie der Massenspektrometrie, paramagnetischen oder Flüssigelektrolytsensoren, stellen sie eine immer stärker genutzte Alternative dar [1, 2].

Nach aktuellem Stand der Technik in der Atemgasanalyse werden die $\mathrm{O}_{2}$-Sensoren gemeinsam mit anderen Messaufnehmern $\left(\mathrm{CO}_{2}-\right.$ Sensoren, Druck- und Temperatursensoren) in einem externen Gehäuse untergebracht. Die Bestimmung des $\mathrm{O}_{2}$-Anteils der Atemluft erfolgt daher ex-situ, indem über einen Probenschlauch kontinuierlich ein kleiner Teil der durch eine Maske oder einen Tubus ventilierten Atemgase abgesaugt und zum Sensor transportiert wird. Diese, als Nebenstromverfahren bezeichnete Messstrategie, birgt jedoch viele potentielle Fehlerquellen, die nur durch hohen technischen und softwareseitigen Aufwand reduziert werden können [2, 3]. Dazu gehören im Wesentlichen: Der gegenüber mechanischen Belastungen anfällige Probenschlauch; die Absaugpumpe, deren beförderte Gasmenge exakt geregelt werden muss; sowie der zeitliche Signalversatz zwischen dem Gas- signal und dem direkt in der Maske erfassten Atemfluss, der in der Spiroergometrie für die Erhebung atemphysiologischer Kenngrößen zusätzlich ermittelt wird.

Ein weiteres Problem aktueller $\mathrm{O}_{2}$-Sensoren sind darüber hinaus die zu hohen Ansprechzeiten, mit denen schnelle Atemgaswechsel (Breath-by-Breath Messung) nicht ausreichend genau aufgelöst werden können. Hier bedient man sich bisher umfangreicher mathematischer Verfahren, mit denen die Signale unter Inkaufnahme zusätzlicher Fehlerquellen künstlich beschleunigt werden [4].

In Anbetracht dieser Herausforderungen wurden am Institut- für Luft und Raumfahrttechnik (ILR) Sensoren aus der Atmosphärenforschung für den Einsatz in der terrestrischen und Weltraummedizin weiterentwickelt $[5,6]$. Dieser $\mathrm{O}_{2}$-Sensoren können aufgrund ihrer robusten, leichten und stark miniaturisierten Bauweise direkt im Hauptatemstrom (in-situ) eingesetzt werden. In Verbindung mit den geringen Ansprechzeiten, die mit diesem neuen Sensor erreichbar sind, besitzt er wesentliche Vorteile gegenüber bisherigen Technologien.

\section{Sensorfunktion und -aufbau}

Das Funktionsprinzip des $\mathrm{O}_{2}$-Sensors lässt sich dem Bereich der elektrochemischen Zelle zuordnen, da er grundlegend aus zwei Elektro- 
den (Anode (A), Kathode $(K)$ ) und einem, beide Elektroden verbindenden, Elektrolyt aufgebaut ist. Dieser besteht im betrachteten Fall aus einer Funktionskeramik, mit der Eigenschaft, bei hohen Temperaturen Ionen zu leiten. Betrieben wird er im amperometrischen Modus (Abb. 1). Dabei wird den Elektroden eine Spannung $\left(U_{E}\right)$ aufgeprägt, die unter hoher Temperatur zur lonisierung des molekularen Umgebungssauerstoffs an der Kathodenseite führt. Die entstehenden $\mathrm{O}_{2}$-lonen bewegen sich über Gitterdefekte durch den Elektrolyt und rekombinieren an der Anodenseite unter Abgabe von Elektronen, die als Sensorstrom $\left(I_{S}\right)$ messbar sind. Dieser ist nach dem Faraday'schen Gesetzt (GI.1) proportional zum transportierten $\mathrm{O}_{2}$-Strom $\left(\mathrm{N}_{\mathrm{O} 2}^{\prime}\right)$ und damit zur Sauerstoffkonzentration in der Umgebung [7]

$I_{S}=\frac{F \cdot z}{N_{A}} \cdot \dot{N}_{O 2}$.

Begrenzt wird der umgesetzte Teilchenstrom durch eine zusätzliche Diffusionsbarriere auf der Kathode, mit der sich über einen angestrebten Messbereich eine lineare Abhängigkeit des Sensorstroms zur ambienten $\mathrm{O}_{2}$ Konzentrentation $\left(\mathrm{C}_{\mathrm{O} 2}\right)$ erreichen lässt $(\mathrm{Gl} .2)$

$$
I_{S}=z \cdot F \cdot A_{\text {diff }} \cdot D \cdot \frac{c_{O 2}}{x_{\text {diff }}} .
$$

Das eigentliche Sensorelement hat dabei eine quadratische Seitenlänge von lediglich $2,5 \mathrm{~mm}$ und wiegt nur wenige $\mathrm{mg}$ (Abb. 2 rechts). Die Fertigung erfolgt durch Dickschichttechnik im Multilayerverfahren, bei der die einzelnen Metall und Keramikschichten Schritt für Schritt aufgetragen werden. Der so ebenfalls auf das Trägersubstrat aufgebrachte Heizer sorgt hierbei für die, zur lonenleitung notwendigen Betriebstemperatur im Bereich von $700-800^{\circ} \mathrm{C}$, die im Bereich der Elektrodenfläche so konstant, aber auch so homogen wie möglich gehalten werden muss. An die Umgebung abgegebene Wärmeströme werden daher unmittelbar durch die Versorgungsleistung des Heizers ausgeglichen. Als Regelgröße dient dabei der Widerstand der Heizwendel.

Durch forcierte Konvektion während der Atmung lokal abgeführte Wärmemengen können allerdings zu stark inhomogenen Temperaturverteilungen führen. Aus diesem Grund werden die Sensorelemente in ein schützendes Gehäuse eingebracht (Abb. 2 links), in dem sie parallel zur Strömungsrichtung ausgerichtet sind. Gleichzeitig dient das Gehäuse als Schutz vor äußeren mechanischen Einflüssen, größeren Partikeln oder Flüssigkeitströpfchen, die mit der Atemluft exhaliert werden. Ebenso sorgt es für die thermische Isolierung nach außen, indem das Element mit Hochtemperaturkeramikkleber auf einen Trägersockel im inneren des PEEK-Gehäuses aufgebracht ist. Um einen schnellen Gasaustausch zu gewährleisten, ist in die Sensorkappe ein großflächiges Schutzgitter eingebracht, das durch seine feinen Maschen gleichzeitig verhindert, dass im Schadensfall abgelöste Sensorfragmente in den Atemstrom geraten. Die elektrische Kontaktierung des Elements erfolgt durch eine Bondverbindung zwischen den Kontaktpins und den Kontaktstiften, die auf der Rückseite des Gehäuses, mit einem Stecker verbunden werden.

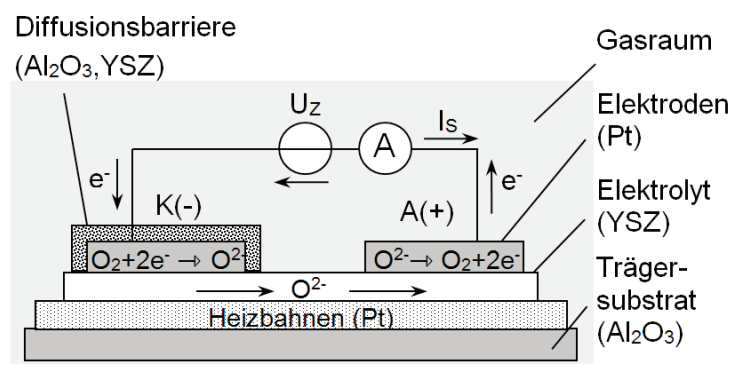

Abb.1: Schematischer Aufbau des $\mathrm{O}_{2-}$ Sensorelements

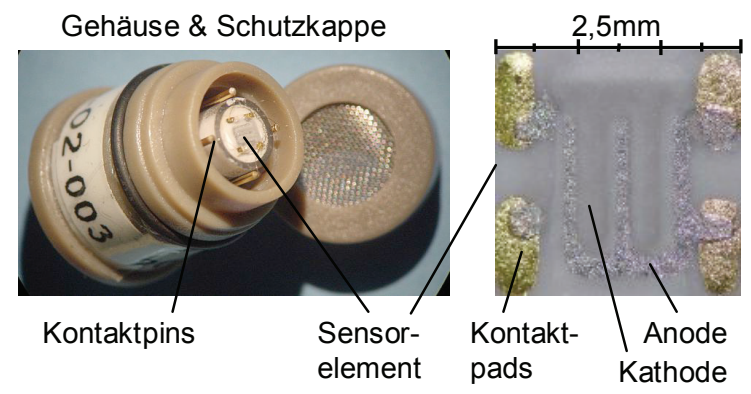

Abb.2: Links: Sensorelement integriert im Gehäuse; Rechts: Mikroskopaufnahme eines $\mathrm{O}_{2}$-Elements

\section{Messmethoden}

\section{Elektronik}

Zur Steuerung der Sensoren, wie zur Erfassung und Verarbeitung der analogen Sensorsignale wird eine am ILR entwickelte Messelektronik verwendet. Sie beinhaltet einerseits die notwendigen Heizerstufen und Regelglieder, um die Sensoren auf die notwendige Betriebstemperatur zu erwärmen und dort konstant zu halten. Zum anderen versorgt sie den $\mathrm{O}_{2}$-Sensor mit der erforderlichen Zellspannung $U_{E}$, die für Standardanwendungen zwischen 0,7 - $1 \mathrm{~V}$ liegt. 


\section{Gasteststand}

Für die Charakterisierung des Sensors hinsichtlich seiner Messgenauigkeit wird ein Gasteststand aus mehreren Massenflussreglern (Bronkhorst, Typ EL-Flow) genutzt, mit dem, unter Verwendung technisch reiner Gase definierte Gemische und Volumenströme generierbar sind. Für ein 2-Komponenetengemisch (z.B. $\mathrm{O}_{2}, \mathrm{~N}_{2}$ ) können damit Regelgenauigkeiten der jeweiligen Gaskomponente von $\pm 0,1 \mathrm{Vol} . \%$ erreicht werden. Neben der Einstellung fester Werte bietet der Teststand zudem die Möglichkeit, Konzentration-FlussProfile zu erstellen, die automatisiert durchlaufen werden. Die Gasbeaufschlagung der Sensoren erfolgt in einer speziellen Messkammer, die eine reproduzierbare Überströmung gewährleistet.

\section{Ansprechzeitenteststand}

Zur Messung der Ansprechzeiten wird ein Teststand verwendet, der ein schnelles Umschalten zwischen zwei Prüfgasen ermöglicht. Realisiert wird dies über Magnetventile (V114A-5G, Firma SMC), die mit einer Schaltzeit von weniger als $5 \mathrm{~ms}$ die entsprechende Gaskomponente ab- oder zuschalten. Sowohl die durchspülte Testkammer, als auch die zuführenden Gasschläuche weisen dabei zusammen lediglich einen Totraum von weniger als $0,7 \mathrm{ml}$ auf und gewährleisten dadurch, über den Transportweg gleichbleibend scharfe Gasflanken.

\section{Messergebenisse}

\section{Kennlinie \& Messgenauigkeit}

Die Erfassung der Kennlinie am gewählten thermischen Arbeitspunkt erfolgt über die Messung des Sensorstroms $I_{S}$ in Abhängigkeit eines, über den Gasteststand applizierten $\mathrm{O}_{2-}$ Stufenprofils $\left(\right.$ Rest $\mathrm{N}_{2}$ ). Aus der Kennlinie lässt sich unter der Wahl geeigneter Stützpunkte eine lineare Kalibrierkurve erstellen und so auf die Messgenauigkeit des untersuchten Sensors schließen. Um zusätzlich Aussagen zur Reproduzierbarkeit und zum Hystereseverhalten des Sensorsignals zu erhalten, werden die Profile zyklisch wiederholt. Abbildung 3 veranschaulicht exemplarisch dazu den Kennlinienverlauf zweier Sensoren $A$ und $B$ über den gesamten $\mathrm{O}_{2}$-Messbereich.

Es ist zu erkennen, dass beide Sensoren eine hohe Nullpunktlinearität bis $100 \% \mathrm{O}_{2}$ aufweisen. Der Nichtlinearisierungsfehler (Toleranzbandmethode) beträgt bezogen auf den Messbereichsendwert (ME) weniger als $1 \%$. Auch die Hystereseabweichung kann für die dargestellten Kennlinien mit 1,5\% v.ME angegeben werden.
Je nach Anwendungsgebiet in der diagnostischen Atemgasanalyse sind unterschiedliche Messbereiche (z.B. Beatmungstechnik 21$100 \%$; Spiroergometrie 10-21\%) interessant, anhand derer die Kalibrierfunktionen angepasst werden. Als Stützpunkte dienen die Gasmesswerte bei Umgebungsbedingungen $(\approx$ $21 \%$ ) und $10 \%$ bzw. $100 \% \quad \mathrm{O}_{2}$-Kalibriergas. Unter dieser Voraussetzung ergibt sich für den Messbereich von 10-21\% eine Messabweichung beider Sensoren von maximal $\pm 0,15$ Vol. $\%$. Für den Bereich von 10-100\% steigt der Fehler auf maximal 3\% v.ME.

Beispielhaft dazu zeigt Abbildung 3 (links) den kalibrierten Signalverlauf $\left(\mathrm{O}_{2}\right.$-Mess) von Sensor $\mathrm{B}$, im Vergleich mit den vorgegebenen $\mathrm{O}_{2-}$ Sollwerten des Gasteststands. Wie die Messgenauigkeit vermuten lässt, kann über den gesamten Messbereich eine hohe Übereinstimmung beider Verläufe erzielt werden.

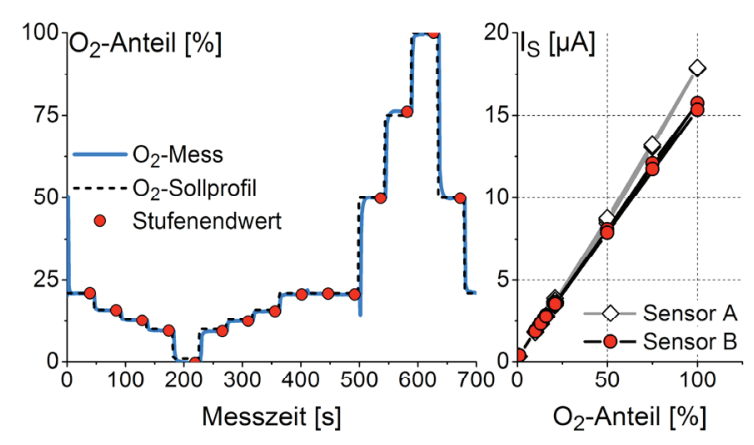

Abb.3: Links: Verlauf des mit Sensor $B$ gemessenen $\mathrm{O}_{2}$-Signals im Vergleich mit dem Sollwertprofil; Rechts: Kennlinie der Sensoren $A$ \& $B$ über den gesamten $\mathrm{O}_{2}$-Messbereich

\section{Ansprechzeit}

Die Ermittlung der Ansprechzeit erfolgt über das mehrmalige Umschalten zwischen Druckluft (ambiente Gaszusammensetzung) und einem, der Ausatemkonzentration äquivalenten Referenzgas $\left(16 \% \mathrm{O}_{2} ; 5 \% \mathrm{CO}_{2} ; 79 \% \mathrm{~N}_{2}\right)$. Als Vordruck wurde 1 bar gewählt, was zu Strömungsgeschwindigkeiten von umgerechnet $35 \mathrm{~m} / \mathrm{s}$ am Einlass der Testkammer führt. Ein Beispielverlauf des, auf den $\mathrm{O}_{2}$-Anteil umgerechneten Sensorsignals ist in Abbildung 4 (oben) dargestellt. Für die Charakteristik des Sensors ist hierbei sowohl die Anstiegszeit $\left(t_{R}\right)$, als auch die Abfallzeit $\left(t_{F}\right)$ von Interesse. Sie beschreiben die Zeitspanne, die das Sensorsignal benötigt, um nach dem Sprung des Eingangssignals von $10 \%$ des Messwerts auf $90 \%$ anzusteigen bzw. abzufallen.

Tabelle 1 fast die Ergebnisse von zwei getesteten Sensoren zusammen. Die Angabe der darin aufgeführten Zeiten erfolgt in Mittelwert 
plus Standardabweichung $\sigma$, errechnet über die jeweiligen 10 Schaltzyklen pro Messung. Darüber hinaus veranschaulicht Abbildung 4 (unten) exemplarisch den normierten Signalabfall eines einzelnen Umschaltzyklus.

Die Daten lassen erkennen, dass für beide Sensoren Ansprechzeiten $\left(t_{R} \& t_{F}\right)$ unter $120 \mathrm{~ms}$ (Sensor A), für Sensor B sogar unter $80 \mathrm{~ms}$, erreicht werden. Der Unterschied zwischen $t_{R}$ und $t_{F}$ fällt vergleichsweise gering aus und ist hauptsächlich auf Messunsicherheiten zurückzuführen. Der Einfluss der Sensorkappe und dem damit erzeugten zusätzlichen Totraums ist bei den gewählten Druckverhältnissen ebenfalls gering, tendiert erwartungsgemäß aber zu höheren Zeiten mit Kappe. Weitere Untersuchungen unter geringeren Vordrücken zeigten einen wesentlich deutlicheren Effekt.
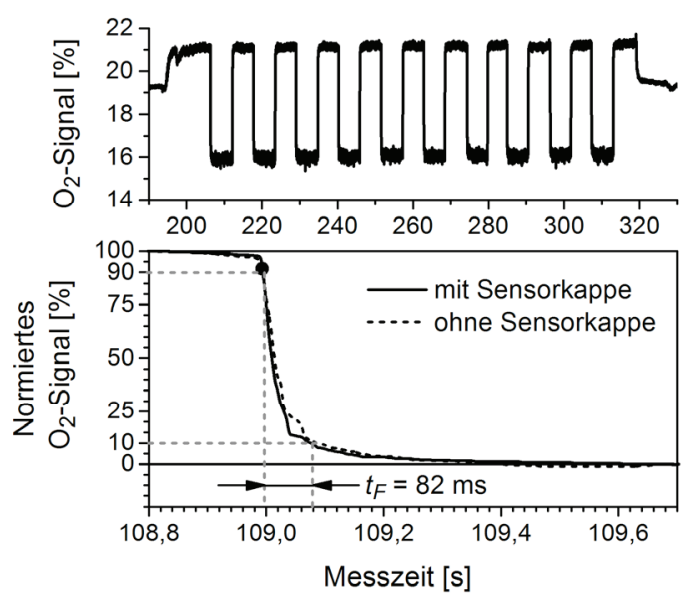

Abb.4: Oben: $\mathrm{O}_{2}$-Verlauf während des Umschaltens zwischen Druck- und Referenzgas; Unten: Beispiel einer abfallenden Gasflanke

Tab.1: Anstiegs- und Abfallzeit zweier Sensoren in Abhängigkeit der Verwendung einer Sensorkappe

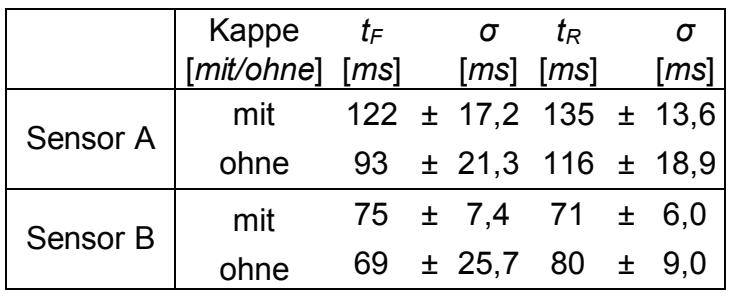

\section{Schlussfolgerungen}

Basierend auf den gewonnenen Ergebnissen kann festgehalten werden, dass der entwickelte $\mathrm{O}_{2}$-Sensor nicht weit von der Messgenauigkeit alternativer Sensoren/ Systeme entfernt ist, die in der Ergospirometrie bzw. der Beatmung zum Einsatz kommen. Als Richtlinie gilt hier die DIN EN 80601-2-55 der Beatmungstechnik, in der ein maximaler Fehler von 2,5 Vol. $\% \pm 2,5 \%$ v.M angegeben ist, bzw. die in der ATS/ACCP vorgeschlagene maximale Abweichung von $1 \%$ v.M für Spiroergometriesysteme [8]. In Letzterem wird zudem die maximale Ansprechzeit angegeben, die für Breath-by-Breath Systeme unter $100 \mathrm{~ms}$ liegen sollte. Verglichen damit und mit dem hohen Grad der Miniaturisierung, die einen Einsatz im Hauptatemstrom erst ermöglicht, weist der Sensor sogar erhebliche Vorteile gegenüber konkurrierenden Sensortechnologien auf.

Einschränkend ist zu sagen, dass in der jetzigen Entwicklungsphase nur ausgewählte Sensoren die dargestellte Messqualität erreichen. Ein Hauptproblem ist die zu geringe Reproduzierbarkeit in der Herstellung der Elemente, die eine zu große Streuung der Sensoreigenschaften mit sich führt. Ebenso ist der, für Festkörperelektrolytsensoren typische Signaldrift eine Herausforderung für zukünftige Entwicklungsschritte. Bisher benötigen die Sensoren eine Mindestbetriebszeit von ca. 48$72 \mathrm{~h}$, bis eine ausreichend hohe Signalstabilität erreicht wird.

\section{Literaturnachweis}

[1] R. Kroidl, S. Schwarz, B. Lehnigk, J. Fritsch, Kursbuch Spiroergometrie, Stuttgart: Thieme Verlag, 2014

[2] R. Kramme, Medizintechnik, Heidelberg: Springer Medizin Verlag, 2007

[3] N. Proctor, K. Beck, J. Appl. Physiol., 81, 24952499, 1996

[4] A.D. Farmery, C.E.W. Hahn, J. Appl. Physiol., 89, 581-589, 2000

[5] S. Fasoulas, R. Baumann, R.M. Glaeser et al.: Solid state electrolyte sensors for the determination of oxygen, carbon dioxide, and total flow rates associated to respiration in human subjects. Executive Summary to the ESA ESTEC Contract No 15450/01/NL/JS CCN, 2006

[6] S. Fasoulas, T. Schmiel, R. Bauman et al.:New miniaturized and space qualified gas sensors for fast response in situ measurements Proc. 40th Int. Conf. on Environmental Systems AIAA 2010-6147 (Barcelona) pp 889-904, 2010

[7] H. Ullmann: Keramische Gassensoren. Akademie Verlag, 1993

[8] ATS/ACCP: "Statement on Cardiopulmonary Exercise Testing," Am J Respir Crit Care Med, 167, 211-277, 2003 